\title{
Archéopages
}

Archéopages Archéologie et société

$41 \mid$ 10/2014-01/2015

La place du cheval

\section{Le cheval dans le château}

L'exemple du château du Guildo aux XI ${ }^{\mathrm{e}}-\mathrm{XVI}^{\mathrm{e}}$ siècles

Horses in the castle. The case of Guildo Castle in the eleventh to sixteenth century $A D$

El caballo en el castillo. El ejemplo del castillo de Guildo en los siglos XI al XVI

Laurent Beuchet

\section{(2) OpenEdition}

Journals

Édition électronique

URL : https://journals.openedition.org/archeopages/951

DOI : 10.4000/archeopages.951

ISSN : 2269-9872

Éditeur

INRAP - Institut national de recherches archéologiques préventives

Édition imprimée

Date de publication : 1 août 2015

Pagination : 20-29

ISSN : $1622-8545$

\section{Référence électronique}

Laurent Beuchet, "Le cheval dans le château », Archéopages [En ligne], 41 | 10/2014-01/2015, mis en ligne le 01 janvier 2017, consulté le 21 janvier 2022. URL : http://journals.openedition.org/ archeopages/951 ; DOI : https://doi.org/10.4000/archeopages.951 


\title{
Le cheval dans le château L'exemple du château du Guildo aux x|e-xv| siècles
}

\author{
Laurent Beuchet Inrap, UMR 6566 «CReAAH»
}

\section{0}

1. Ce site a fait l'objet de recherches importantes de 1994 à 2013, financées par l'État et le conseil général des Côtes-d'Armor, propriétaire du site, sous la direction de Laurent Beuchet, Inrap.
Le château du Guildo (Côtes-d'Armor) est situé au fond de la profonde ria de l'Arguenon, sur la côte nord de la Bretagne ${ }^{\mathbf{1}}$. La fouille exhaustive du monument a mis au jour quatre espaces interprétés comme ayant accueilli des chevaux, depuis la première occupation castrale aux $\mathrm{XI}^{\mathrm{e}}-\mathrm{XII}^{\mathrm{e}}$ siècles jusqu'à la remise en défense $\mathrm{du}$ château pour les guerres de la Ligue à la fin du $\mathrm{XVI}^{\mathrm{e}}$ siècle (Beuchet, à paraître).

\section{Une écurie intégrée à la demeure seigneuriale} du premier château

Aucune source documentaire, directe ou indirecte, ne mentionne le château du Guildo avant le XIV ${ }^{\mathrm{e}}$ siècle. Pourtant, la fouille a mis en évidence les vestiges arasés d'un établissement fortifié, sans doute construit dès le $\mathrm{XI}^{\mathrm{e}}$ siècle dans le contexte des luttes entre les comtes de Bretagne et les héritiers de l'ancienne vicomté d'Alet (Beuchet, 2012). Au nord de la cour actuelle, le plan d'un vaste bâtiment (environ $5 \mathrm{~m} \mathrm{x} 18 \mathrm{~m}$ ), de construction mixte de maçonnerie et de charpente, a été reconnu [ill. 1a].

Une cloison marque une séparation du bâtiment en deux espaces. Les sols de terre battue de la pièce occidentale livrent quelques fragments de céramique culinaire datables de la fin du $\mathrm{XI}^{\mathrm{e}}$ siècle au début du XIII ${ }^{\mathrm{e}}$ siècle indiquant une fonction domestique de cet espace. La seconde pièce, à l'est, présente un plan incomplet marqué par des fondations de poteaux. Sa longueur peut atteindre 7,50 m environ. L'épaisseur des maçonneries, mais surtout l'importance des fosses de fondation de poteaux permet de restituer la présence d'un étage sur tout ou partie de la construction. Un léger surcreusement de la roche, large de $3 \mathrm{~m}$ environ, est visible dans l'espace principal et a piégé quelques vestiges de sols de limons et d'arène. Sa fouille a livré de petits éléments de décor de vêtement ou d'accessoires (ceinture, baudrier, harnachement, etc. [ill. 1b]), réalisés en alliage cuivreux, largement présents sur les sites médiévaux entre le XII ${ }^{\mathrm{e}}$ et le XIV ${ }^{\mathrm{e}}$ siècle. Ni leur morphologie, ni leur décor ne permet malheureusement d'affiner cette chronologie. Deux fragments de fers d'équidés ont également été recueillis. Ils présentent des rives ondulées et des étampures allongées [ill. 1c], éléments qui tendent à disparaître au cours du XIII ${ }^{\mathrm{e}}$ siècle et qui sont souvent considérés comme signes d'une certaine ancienneté. Ils accompagnent une boucle rectangulaire, généralement interprétée comme boucle de sous-ventrière, très fréquente dans les habitats aristocratiques des $\mathrm{X}^{\mathrm{e}}$ et $\mathrm{XI}^{\mathrm{e}}$ siècles (Bourgeois, 2009, p. 234-235). Ces éléments architecturaux et mobiliers permettent de restituer, à la pointe de l'éperon fortifié, un bâtiment à un ou plusieurs étages, dont le rez-decour est occupé à l'ouest par un espace domestique. À l'est, la forte représentation de mobilier métallique en rapport avec l'équipement du cheval ou du cavalier laisse supposer la présence d'une écurie.

\section{Un ensemble dédié au cheval au $\mathrm{Xv}^{\mathrm{e}}$ siècle}

Un château de pierre est construit au milieu du XIII ${ }^{\mathrm{e}}$ siècle pour remplacer l'enceinte de terre. Au milieu du $X^{e} v^{e}$ siècle, le château fait à nouveau l'objet d'importants travaux. Les communs, adossés à la courtine ouest, sont transformés et complétés par une écurie et une forge maréchale (Beuchet, à paraître). L'écurie, construite en maçonnerie, présente un plan rectangulaire [ill. 2]. Elle s'organise en deux espaces symétriques, de part et d'autre d'un mur de refend central. De chaque côté, un couloir dessert depuis la cour une pièce dont il est séparé par une cloison de bois. Le 


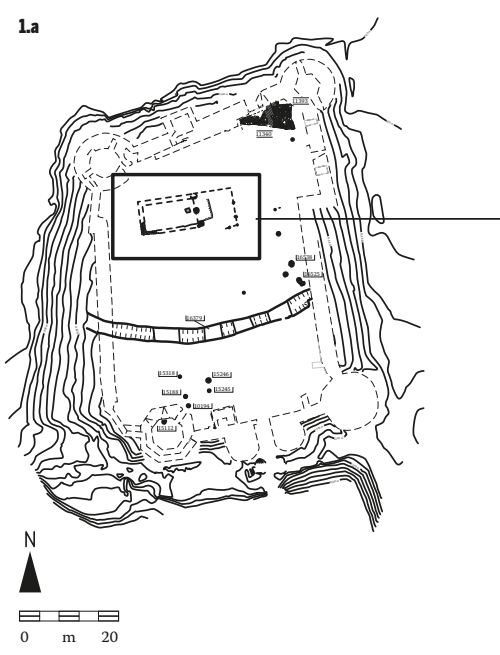

\section{1.a. Le mobilier métallique associé à la première résidence seigneuriale du Guildo (xle-xlre siècle) a permis d'interpréter la fonction des espaces à l'intérieur du grand ensemble; \\ 1.b. Boucles et décors de \\ lanières, alliage cuivreux; \\ 1.c. Fers à sabot et boucle \\ de harnais, fer.}

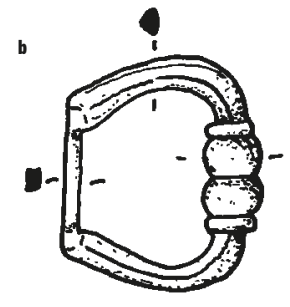

$12361 / 3$
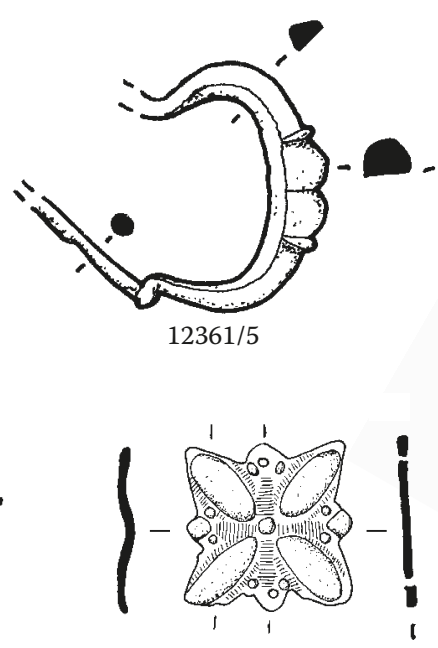

$12376 / 3$

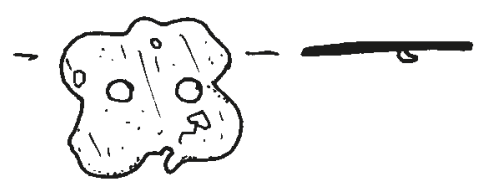

12361/4

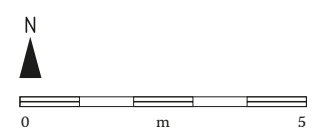

- Vestiges des phases postérieures 118,52 Altimétrie

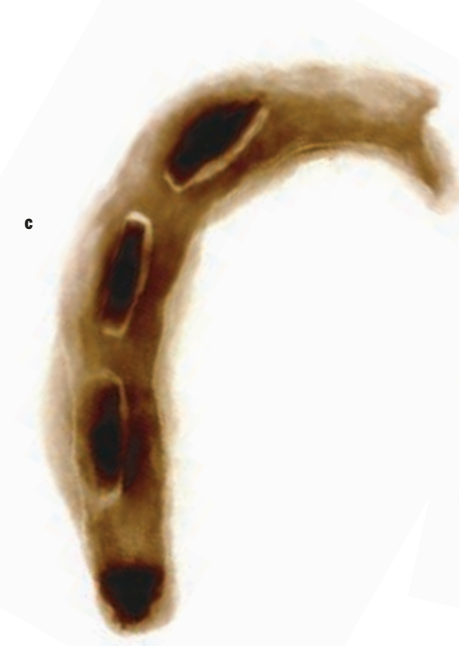

12361/8 5 (radiographie)

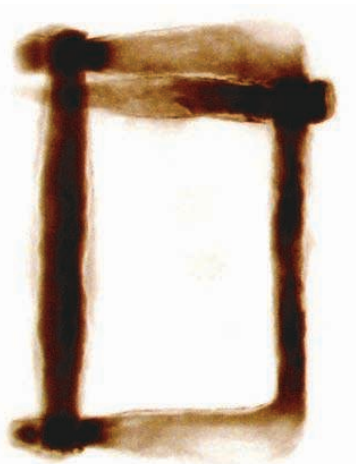

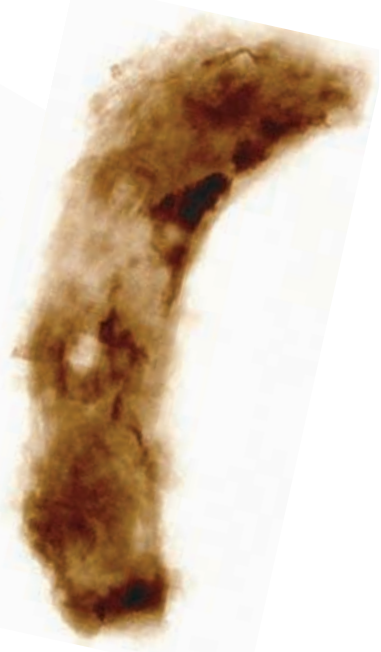

12361/9 (radiographie)
12376/3 (radiographie)

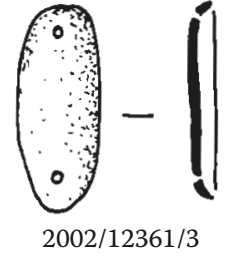

2002/12361/3

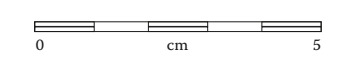

E

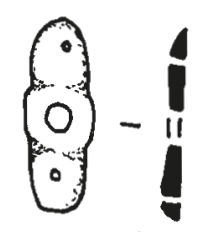




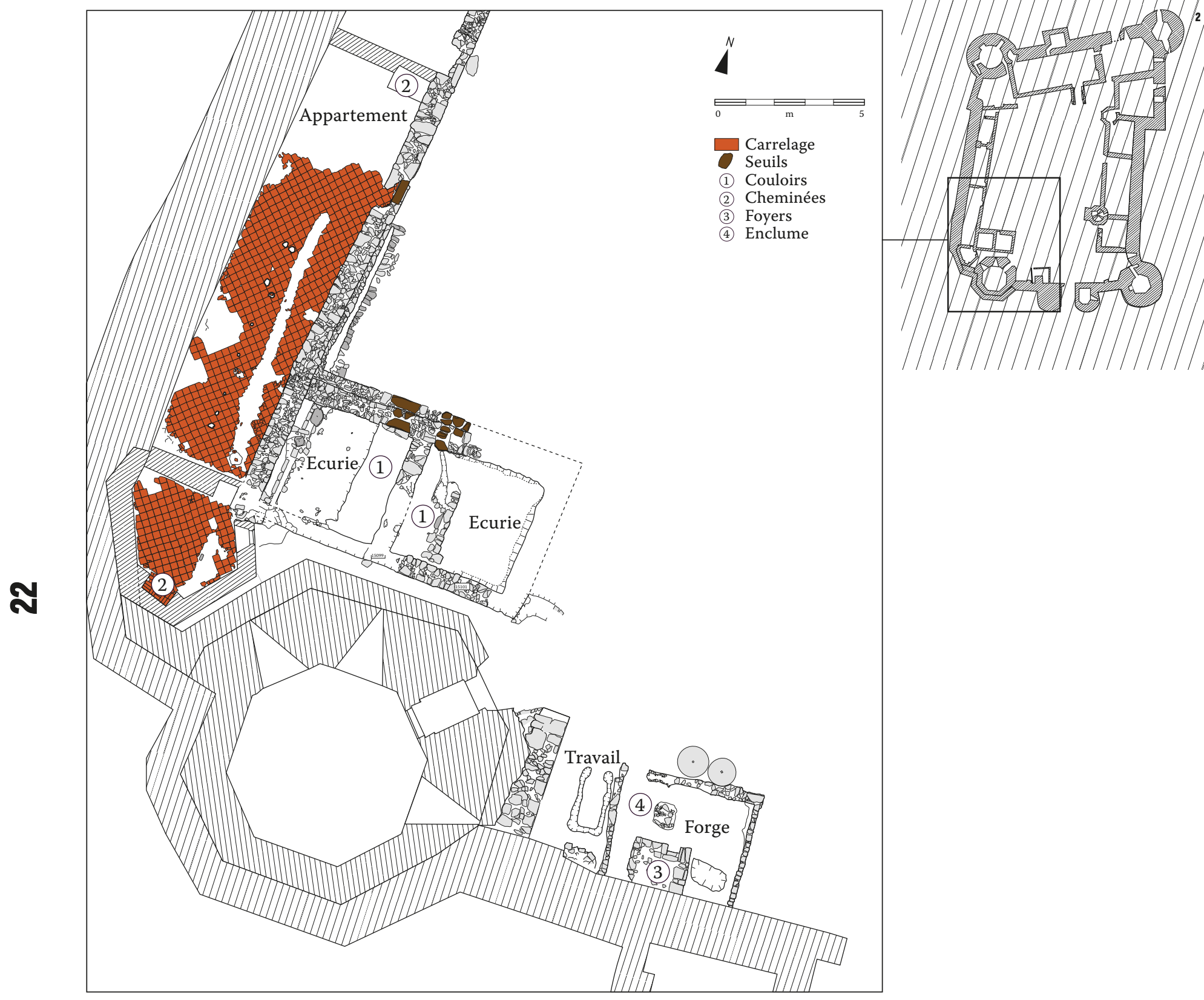

2. L'analyse de la

stratigraphie a montré la

parfaite contemporanéité

de l'ensemble des

installations dédiées au

cheval et à son entretien

au milieu du xve siècle,

datées par un abondant

mobilier, monétaire

notamment.
3. Restitution

architecturale de l'écurie

du milieu du xve siècle.

L'épaisseur assez faible

des murs interdit la

présence d'un étage carré.

La toiture présentait

deux pentes couvertes

d'ardoises d'après

les éléments retrouvés

dans les niveaux

de destruction.

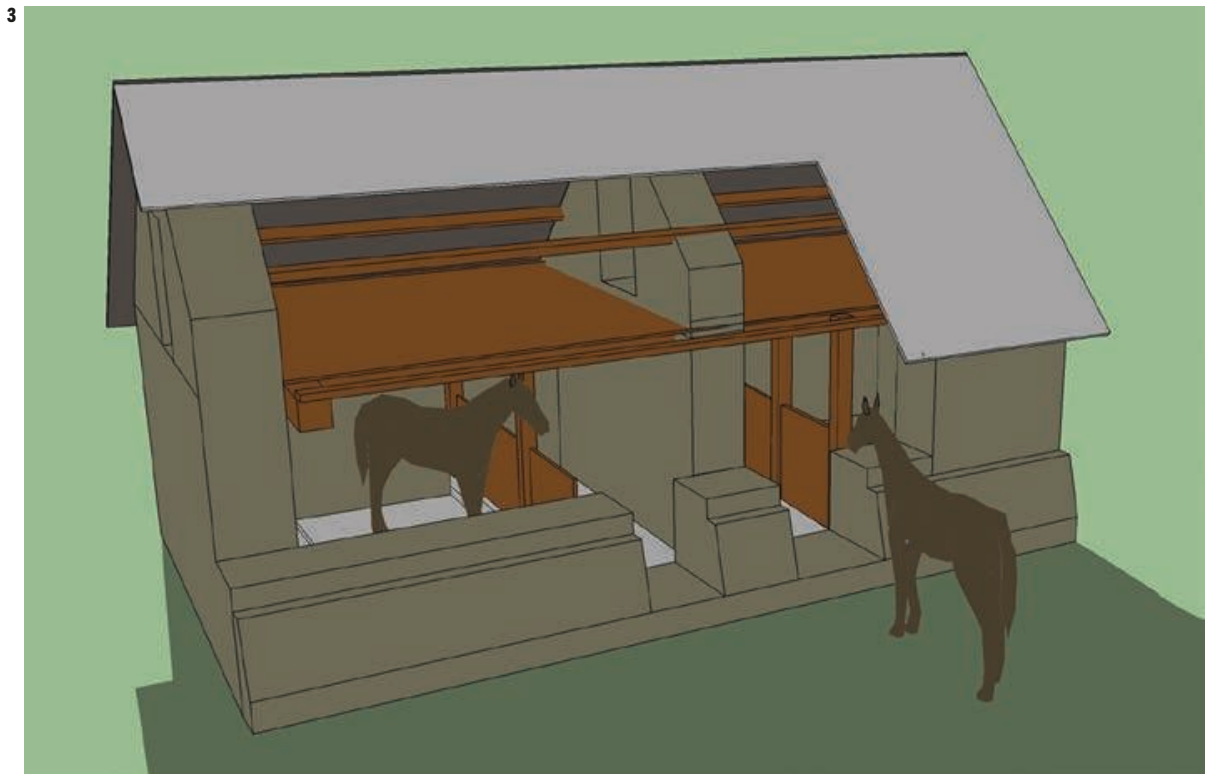


sol de ces pièces, creusé dans le rocher et aplani par une terre battue, est situé légèrement en contrebas du couloir et présente une pente vers un exutoire. Au sud, la façade du bâtiment est doublée par un muret large de o,3o m environ qui se poursuit à la base du mur du commun, à l'ouest. Il présente un fruit important et est doublé à son pied par l'aménagement d'un fil d'eau. Ces vestiges, bien que très arasés, permettent de proposer une restitution du bâtiment [ill. 3]. Il est possible que chaque pièce ait été divisée en stalles ouvertes perpendiculairement au couloir. La largeur du bâtiment permet dans ce cas de restituer au maximum deux stalles par pièce. Toutefois, la largeur du couloir semble trop faible $(1,30 \mathrm{~m})$ pour permettre la rotation du cheval quittant sa stalle. Il semble donc plus probable que chacune des pièces ait été dévolue à un unique box desservi par un couloir qui pouvait également être utilisé pour le rangement des harnachements et de divers équipements. L'état des vestiges ne permet pas de restituer l'emplacement ni le type de mangeoire ou de râtelier. L'aménagement des doublages de maçonneries en pied des murs sur la cour ne semble pas avoir de fonction architecturale particulière. Il est possible qu'il s'agisse de simples murets, sans doute peu élevés, destinés à faciliter la monte. Leur fruit prononcé peut s'expliquer par la volonté d'éviter que les chevaux ne se blessent contre une arête trop marquée. Leur présence peut traduire l'utilisation de l'espace qu'ils bordent, d'une aire de $50 \mathrm{~m}^{2}$ environ, pour le soin ou le travail des chevaux.

À quelques mètres de distance au sud, la fouille a mis au jour les vestiges d'une forge abritée dans un bâtiment adossé à l'arrière du châtelet d'entrée (Beuchet et Picault, 1998) $^{\mathbf{2}}$. La forge proprement dite est installée dans un bâtiment appuyé contre le mur arrière de la tour ouest du châtelet d'entrée. La toiture était constituée d'ardoises, comme l'attestent les niveaux de destruction. On accède à l'intérieur par une porte aménagée près de l'angle nord-ouest du bâtiment dont le sol était aménagé en terre battue. À l'intérieur du bâtiment ont été découverts l'emplacement d'un vaste foyer maçonné et de son cendrier ainsi que l'emplacement d'une enclume. À l'ouest du bâtiment, l'empreinte d'un bâti en bois rectangulaire a été dégagée, constituée de puissants poteaux reliés par des poutres. La taille et la position de cette structure, ainsi que la découverte de très nombreux clous à ferrer sur les sols environnants permettent de replacer ici un «travail » de maréchal-ferrant, dans lequel on entrave les animaux lors du ferrage mais également un espace de soins.

À l'ouest de l'écurie, le bâtiment des communs est une longue construction qui s'adosse sur toute la courtine occidentale [ill. 2]. L'ensemble est fortement rénové au milieu du $\mathrm{Xv}^{\mathrm{e}}$ siècle. Une suite de deux pièces est alors aménagée au sud. L'accès se fait depuis la cour par la pièce nord, d'une surface de plus de $50 \mathrm{~m}^{2}$. Cette grande salle présente pour seul aménagement une cheminée, située sur son refend nord. Son sol constitué de carreaux n'est que partiellement conservé. Il est probable que une à deux baies s'ouvraient à l'est pour éclairer cette pièce. Des traces d'enduits de chaux sont conservées sur les murs est, sud et ouest de la salle. Elle donne accès au sud à une pièce plus petite, au plan hexagonal irrégulier, carrelée de la même façon que la grande salle. Elle est munie d'une cheminée et d'une petite baie ouvrant à l'est sur un délaissé à l'arrière de l'écurie. Ces deux pièces montrent une grande cohérence par leur distribution et leur mise en œuvre. Elles forment ce que l'on peut considérer comme un appartement, muni d'éléments de confort. On note cependant l'absence d'accès à des latrines privatives. Par ailleurs, la position de ce logement à l'extrémité sud des communs, loin du logis seigneurial installé sur les fronts nord et est de la cour, indique que ses occupants n'appartenaient pas à la noblesse, mais jouissaient toutefois d'un statut particulier. On peut supposer, du fait de sa position proche de l'écurie, que ce logement était destiné au personnel en charge de l'entretien et du soin des chevaux.

Les installations et le mobilier [ill. 4] mis au jour dans l'angle du château du Guildo forment un ensemble à ce jour totalement inédit en fouille pour le Moyen Âge. L'écurie frappe par sa modernité. Son aménagement le plus plausible, en deux boxes individuels, ne trouve aucun équivalent dans la documentation disponible, tant archéologique qu'écrite ou iconographique (Schwien et Jeannin, 2012, p. 121-122). Â cette écurie s'adjoint une forge maréchale, qui présente un plan très cohérent et fonctionnel, vraisemblablement ordonné et mis en œuvre par le maréchal lui-même. C'est sans doute pour le logement de ce personnage qu'est aménagé un appartement qui se distingue clairement du reste des communs, à proximité immédiate de l'écurie. On voit en effet, en cette fin du Moyen Âge, apparaître, au service des princes, des maréchaux vétérinaires, véritables spécialistes au service du $\mathrm{cheval}^{3}$. Si l'on adjoint à ces éléments l'espace de cour formé par l'angle des écuries et des communs, destiné aux soins comme semble l'indiquer l'aménagement de murets à la base des façades, c'est une surface de plus de $300 \mathrm{~m}^{2}$ qui est alors dévolue au cheval, soit un dixième de la superficie du château. Le faible nombre d'animaux qu'abrite cette écurie et le grand soin qu'on leur apporte posent question. Il est possible que l'écurie soit réservée aux animaux malades ou aux juments prêtes à mettre bas. Il est également possible qu'elle abrite les étalons loin des juments et des jeunes, ou des bêtes exceptionnelles à qui elle sert d'écrin.

Quoi qu'il en soit, la qualité de l'installation et la présence à demeure d'un personnel dédié s'expliquent par le niveau social élevé des occupants des lieux. Le château est alors la propriété de Françoise de Dinan, jeune orpheline héritant en 1444 des fortunes très considérables

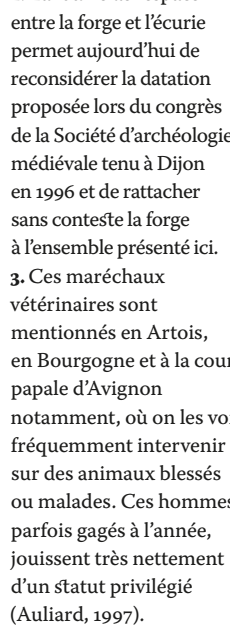

entre la forge et l'écurie reconsidérer la datation de la Société d'archéologie en 1996 et de rattache sans conteste la forge 3. Ces maréchaux mentionnés en Artois, papale d'Avignon 
4. Mobilier métallique

en lien avec le cheval,

issu des niveaux

contemporains de l'écurie

et de ses annexes

au xve siècle.

1 et 2: éperons:

3 et 4 : éléments de mors;

5: étrier;

6: étrille;

7: poinçon de bourrelier.
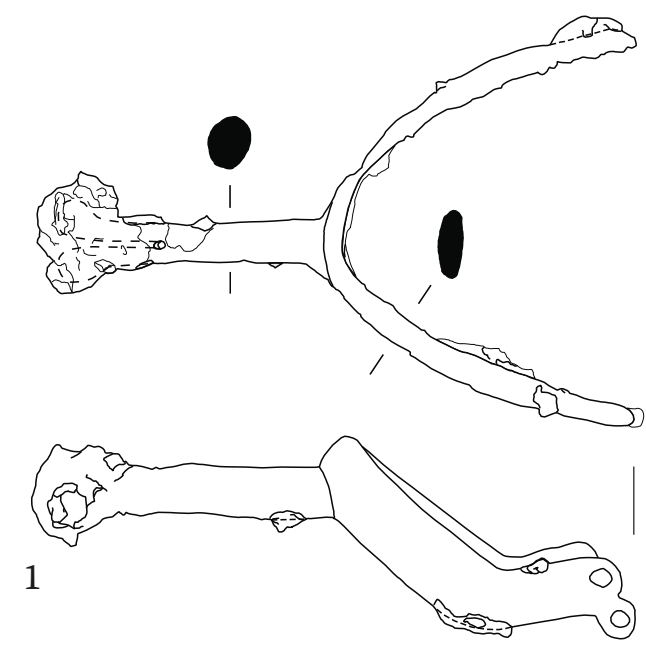

A
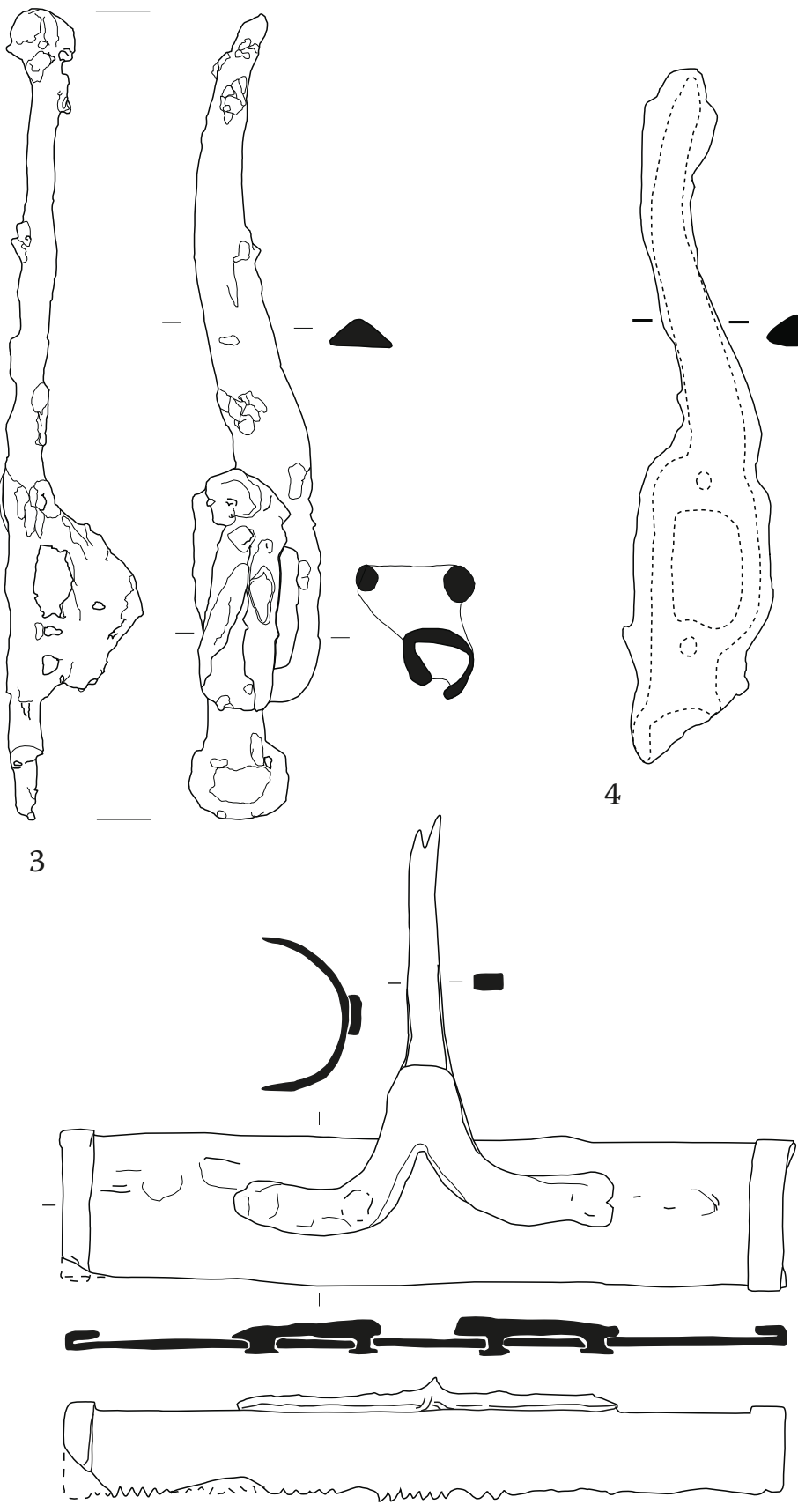

6
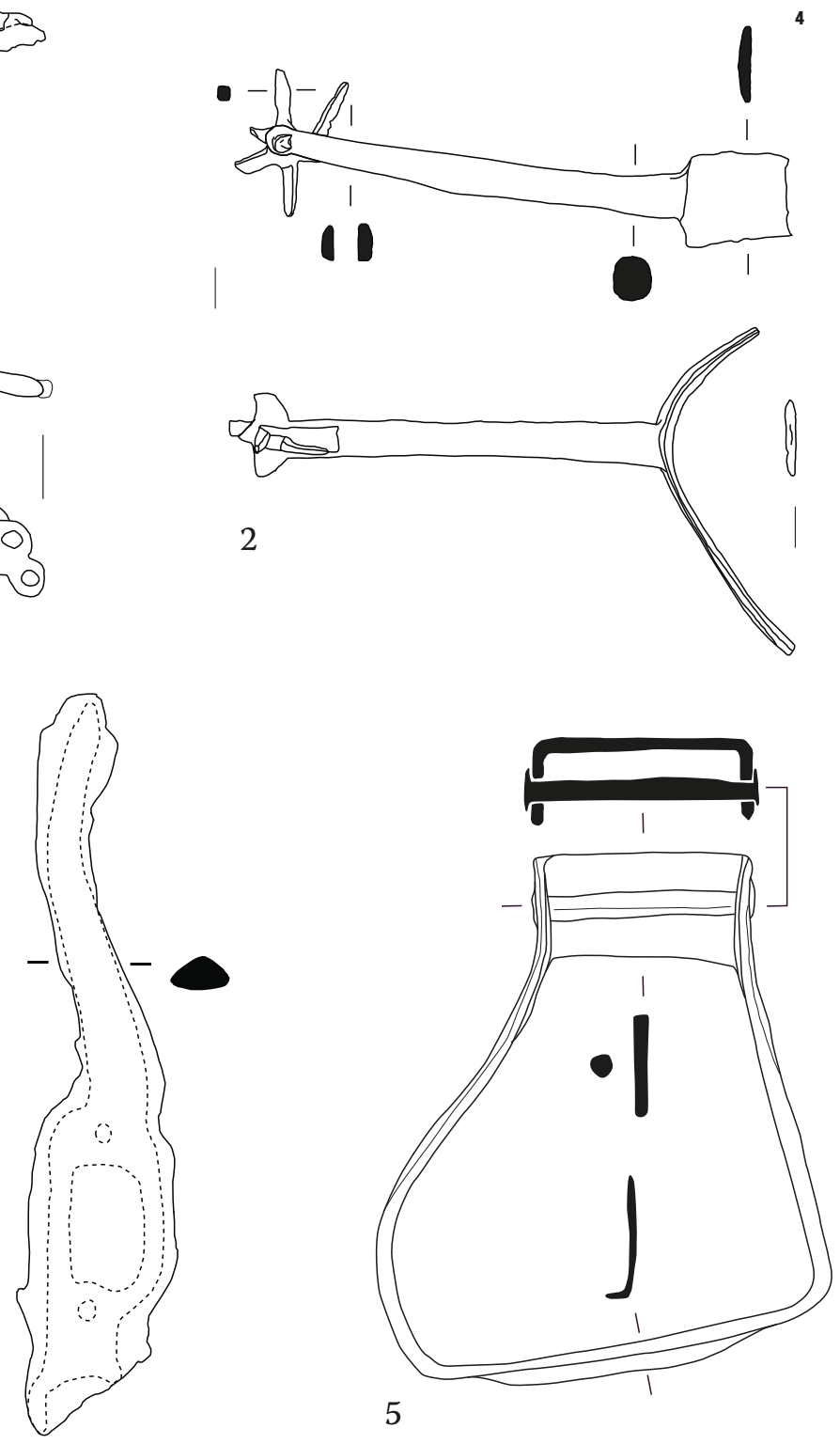

5 
des familles de Dinan et de Beaumanoir. Elle est mariée à Gilles de Bretagne, fils cadet du duc Jean V. Le jeune couple fait du Guildo sa résidence principale qui peut dès lors être considérée comme un véritable petit palais.

\section{Des aménagements hâtifs en temps de guerre}

À partir des années 1460, de vives tensions se font jour entre la France et le duché de Bretagne. La guerre, inévitable, éclate en 1487. Dès le mois de mars de cette même année, le duc François II demande à Jean Henliguen, lieutenant du château du Guildo, de mettre «... a la garde d'iceluy XXIII gentilzhommes et les francs archers... » (Mandement de François II, AD Loire-Atlantique, $2 \mathrm{Mi} 31 / \mathrm{R}_{3}$, fol. 122). C'est sans doute dans ce cadre que le grand cellier, qui occupe les soubassements de la grande salle du château, est transformé en écurie [ill. 5]. Il est accessible depuis la cour par un large escalier, très doux, taillé dans la roche, qui a été réaménagé lors des travaux du milieu $\mathrm{du} \mathrm{Xv}^{\mathrm{e}}$ siècle. Une seule meurtrière s'ouvre dans la courtine nord.

Lors des travaux de transformation, un mur de séparation est monté au droit de l'accès à la cave, ménageant à l'ouest un espace de $11 \mathrm{~m}$ de long et à l'est une pièce beaucoup plus réduite (longue de 4 m environ). Cette cloison est simplement collée contre les parements des murs nord et sud. Elle est ouverte en son centre d'une porte et trois percements traversent sa base, au niveau du sol.

Quatre cavités sont pratiquées dans le mur nord de la grande pièce occidentale, par descellement de moellons de la maçonnerie, à une hauteur constante de 1,40 m au-dessus du sol ; elles sont régulièrement espacées les unes des autres, de $2 \mathrm{~m}$ environ. Cet espacement semble un peu large pour permettre l'installation de stalles (Schwien et Jeannin, 2012 ; Dufour, 2013). Elles peuvent en revanche correspondre à la fixation d'un râtelier. D'autres creusements situés à la même altitude dans le mur ouest peuvent marquer le retour de cette installation. Le sol de la cave, constitué du rocher aplani et nivelé par un sol de terre battue, ne semble pas avoir subi de modification. Il ne montre aucune trace permettant de restituer une mangeoire. Une file de faibles impacts signale la présence de poteaux pouvant marquer une séparation dans la pièce, ménageant un couloir de circulation au sud, large de 1,70 m environ. En reprenant les préconisations de $1,50 \mathrm{~m}$ pour chaque cheval, cette disposition permet d'estimer que l'espace pouvait accueillir 7 à 8 chevaux, disposés en une file le long du mur nord du cellier. La pente générale du sol est aménagée vers l'est, où un caniveau draine les eaux de ruissellement provenant de la cour à travers la courtine nord.

Le logis nord a été incendié lors du conflit, probablement en 1490. La fouille des sols conservés sous les niveaux d'incendie, dans la petite pièce à l'est de la cloison et dans la cave attenante occupant l'angle nord-est du château, a livré plus de 4 ooo restes végétaux (Dietsch-
Sellami, 2000 et 2003). La moitié des restes identifiés appartiennent à des céréales, parmi lesquelles l'avoine représente $96 \%$ des espèces déterminées, auprès de l'orge vêtue, du seigle et du blé. La concentration des graines et leur carbonisation incomplète indiquent que celles-ci étaient disposées dans un contenant de bois ou de textile qui les a protégées de l'incendie. Le reste des taxons est très majoritairement composé d'herbacées sauvages, essentiellement issues de prairies, mais également de milieux humides, roselières et marais. Parmi ces restes, les nombreux fragments de tiges permettent de les identifier comme fourrage provenant de prairies de fauche. La présence de ces éléments, fragiles, mais également l'observation de graines de jonc encore soudées suggèrent un dépôt primaire, en place. Il semble donc que ces espaces étaient dévolus au stockage du fourrage, composé de céréales et de foin.

Un aménagement similaire a été mis au jour dans un second cellier, situé au rez-de-cour du logis oriental. Il s'agit d'un espace long de 14,5 m pour une largeur de 6,30 $\mathrm{m}$ [ill. 6]. Une porte donne accès à la cour dans l'angle nord-ouest de la pièce. Ses piédroits ont disparu mais sa largeur peut être estimée à $1 \mathrm{~m}$ environ. Deux jours s'ouvrent sur la cour, ils montrent des fixations de grilles et étaient vraisemblablement pourvus de volets intérieurs. Une canonnière perce la base de la courtine à l'est. La fouille a mis au jour un aménagement tardif, formé par un sol de galets provenant du fleuve, disposés debout ou de chant dans un lit de sable. L'ensemble atteint une épaisseur d'une vingtaine de centimètres. Il n'occupe qu'une partie de l'espace du cellier, au nord de la canonnière. Sa longueur atteint près de $10 \mathrm{~m}$ pour une largeur de 3,40 m. Sa surface, aplanie par l'ajout de sable comblant les interstices entre les galets, marque une pente de 4 à $5 \%$ vers le centre de la pièce. Cet aménagement reprend les dispositions préconisées dans les traités de maréchalerie et d'hippiatrie - et généralement observées dans les écuries contemporaines - de disposer dans les écuries un sol solide permettant l'évacuation des eaux et des urines pour prévenir les infections du pied et du sabot (Schwien et Jeannin, 2012 ; Liévaux, 2005). En revanche, aucun système de collecte n'est présent au bas de la pente pour évacuer ces matières hors de la pièce.

Comme dans le cellier nord, une série de sept creusements a été effectuée dans le parement de la courtine, à une altitude constante, environ $1 \mathrm{~m}$ au-dessus du sol. Leur écartement est moins régulier, l'entraxe variant de $1 \mathrm{~m}$ à $1,60 \mathrm{~m}$. Ils se limitent à l'emprise dessinée par le sol de galets et semblent donc lui être associés. Aucun autre aménagement n'a été observé, en dehors du renforcement du passage vers la porte d'entrée à l'aide de dalles de schiste et de granite. Cette irrégularité dans l'écartement des creusements et l'absence de traces de cloisonnement au sol laissent penser que ces encastrements sont liés ici encore 
5.a

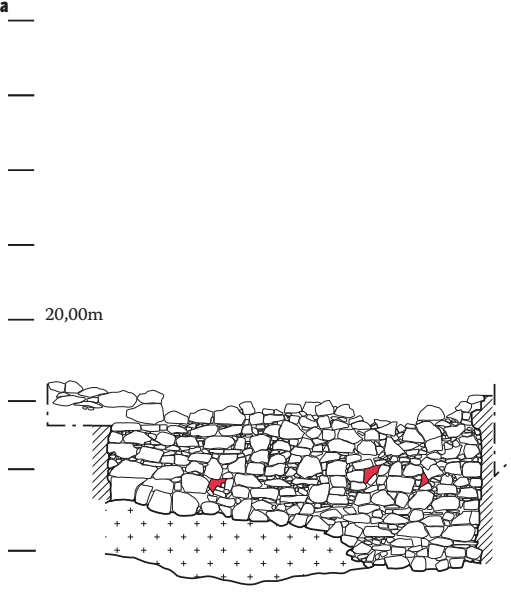

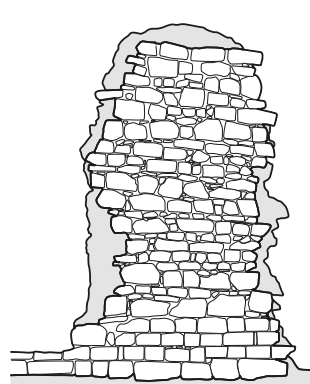

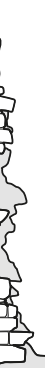

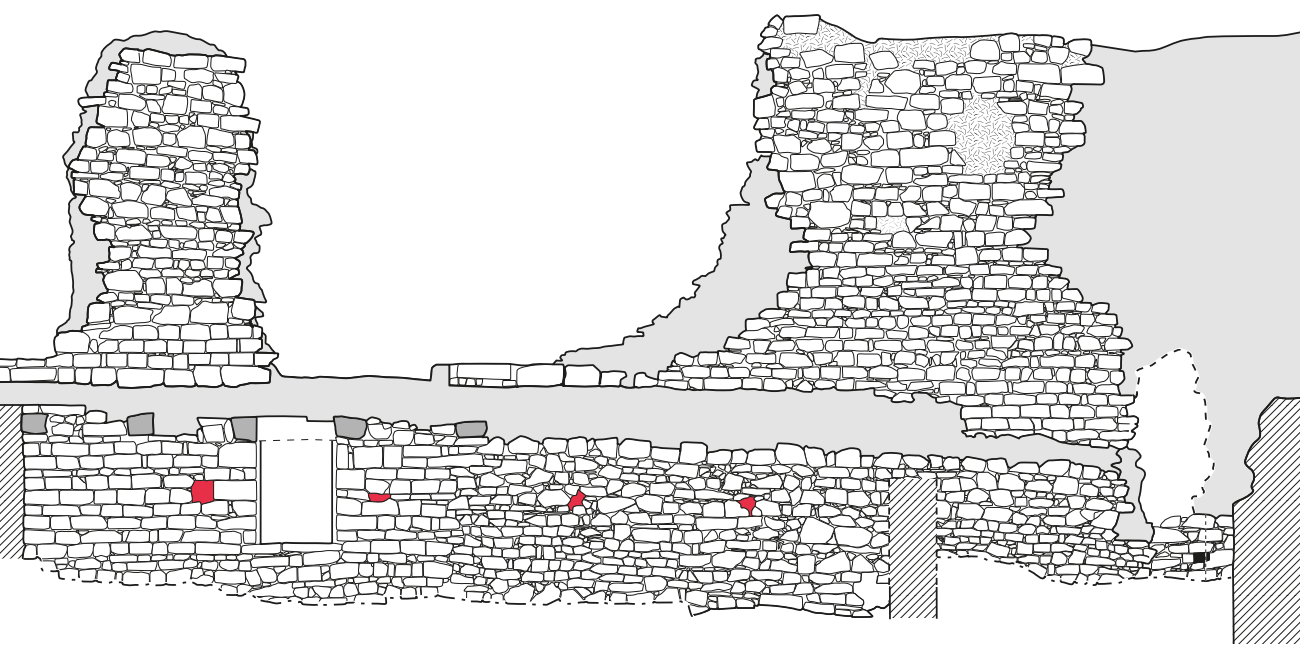

噔谓 Zone non lisible

Parement manquant

ZIA Maçonnerie en coupe

$\square$ Consoles de plancher.

a Bûchages du parement

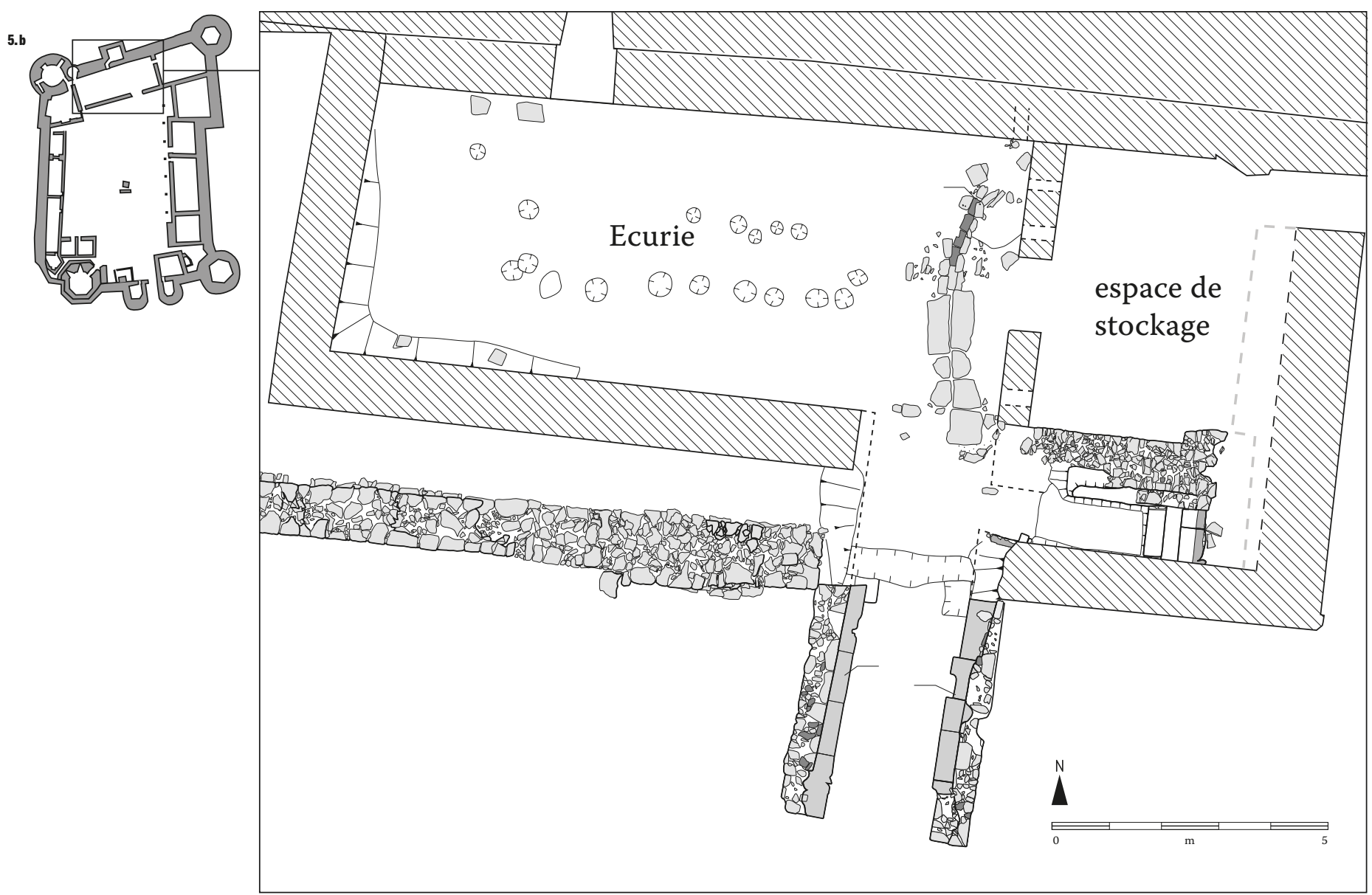




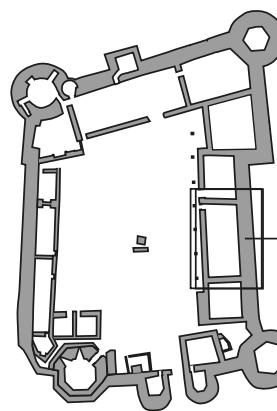

6. Vue du sol de galet posé

pour la transformation

du cellier oriental en

écurie à la fin du xvie siècle.

On remarque les bûchages

dans le parement de la

courtine, correspondant

au scellement d'une

N

mangeoire.

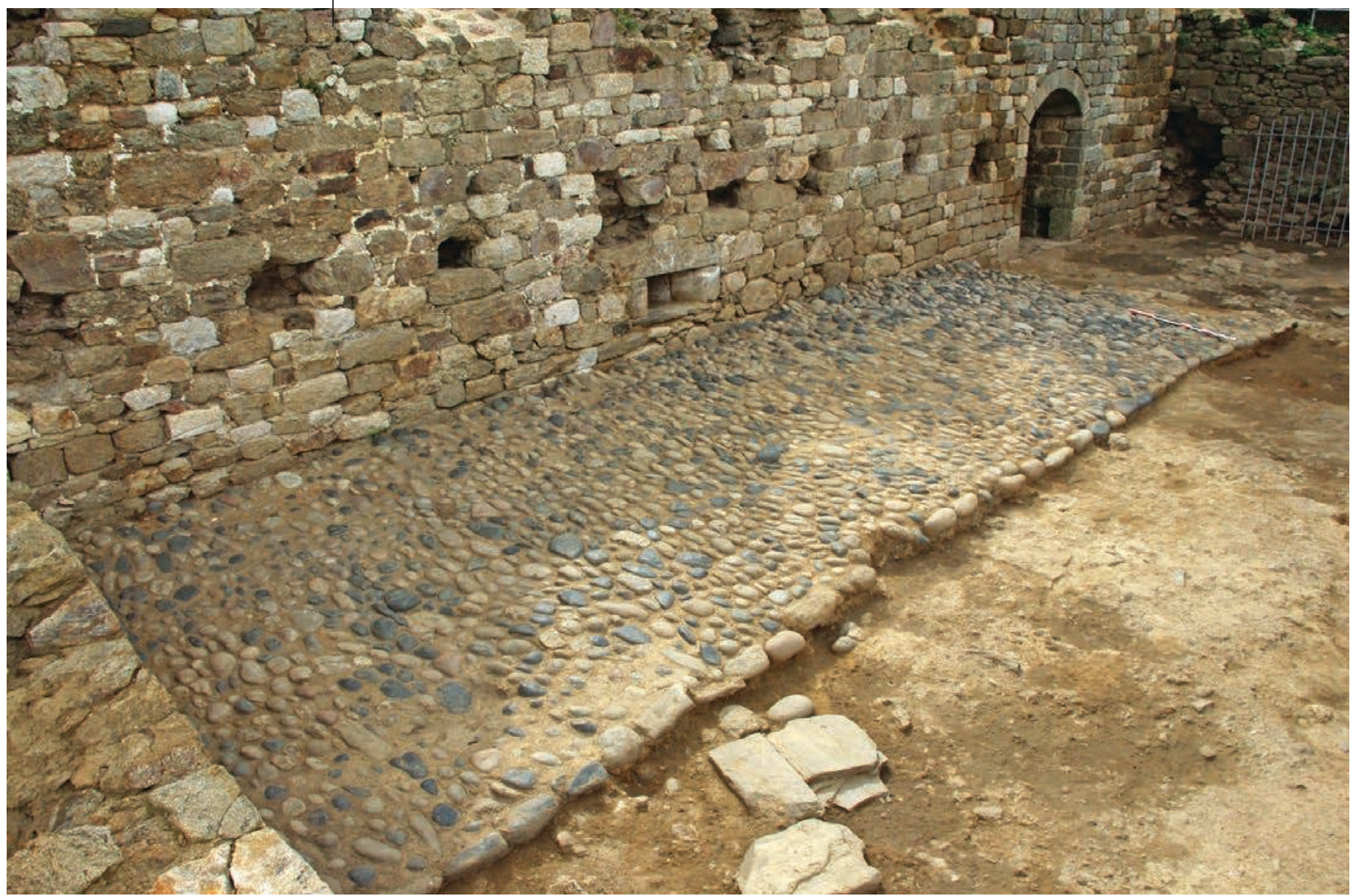


à l'installation d'un râtelier ou d'une mangeoire. En reprenant les espacements préconisés pour chaque cheval, on peut considérer que cette écurie pouvait accueillir six à sept chevaux.

La mise en œuvre de ces aménagements s'inscrit dans un ensemble de travaux datables de la fin du XVI ${ }^{\mathrm{e}}$ siècle et qui correspondent tous à la remise en défense du château lors des guerres de la Ligue de Bretagne, entre 1588 et 1598. La documentation rapporte par ailleurs plusieurs sièges du château durant ce conflit.

Ces deux exemples, bien que non contemporains, montrent des aménagements comparables, réalisés dans des contextes semblables de remise en défense du château. Dans les deux cas, ils traduisent certainement la volonté d'abriter dans la forteresse, hors d'atteinte de l'ennemi, un certain nombre de montures. Ce nombre est limité pour chaque installation à six à huit bêtes. Outre les francs-archers provenant des paroisses avoisinantes, le mandement ordonnant la mise en défense du château en 1487 mentionne 23 gentilshommes. Bien qu'il soit impossible de déterminer le nombre exact de montures dont pouvaient disposer ces personnages et leur accompagnement - on compte généralement pour chaque homme d'armes au moins un écuyer et un page, disposant chacun d'une monture (Contamine, 1986, p. 243) -, il reste certain que la capacité de l'écurie présentée ici devait être bien insuffisante, même en ajoutant l'écurie précédente, toujours active et augmentée hâtivement d'un box supplémentaire. On ignore tout du logement des autres chevaux, et des critères présidant au choix de ceux hébergés dans cette écurie.

L'installation de l'écurie dans le logis oriental à la fin du XVI ${ }^{\mathrm{e}}$ siècle appelle les mêmes remarques. On ne connaît rien des garnisons, qu'elles soient de la Ligue ou de l'armée royale, mises à la garde du Guildo durant les guerres de Religion.

Toutefois, H. Le Goff rapporte l'exemple de M. du Bordage, gentilhomme huguenot qui demande en mai 1593 l'aide anglaise pour fortifier son château, comparable à celui du Guildo. Parmi les pièces d'artillerie et les projectiles, les piques et les hallebardes, apparaissent « vingt harnois complets », témoignant du nombre minimum de chevaux à disposition de la garde (Le Goff, 2010, p. 231-232). Ici encore, le nombre de places restituables dans l'écurie mise au jour par la fouille paraît bien faible en regard du nombre probable de chevaux nécessaires à la garnison du Guildo.

Les quatre écuries découvertes au château du Guildo forment un ensemble exceptionnel d'écuries médiévales et modernes mises au jour par la fouille sur un même site. Ce caractère d'exception tient sans doute principalement à l'exhaustivité de l'étude menée sur ce monument et au manque général de fouilles sur les communs des châteaux. En révélant des vestiges appartenant à quatre phases successives d'aménagement du château, la fouille permet d'aborder plusieurs points de vue quant à la place du cheval dans le château du XI ${ }^{\mathrm{e}}$ au XVI ${ }^{\mathrm{e}}$ siècle et de proposer quelques réflexions.

L'écurie la plus ancienne, datable des $\mathrm{XI}^{\mathrm{e}}$ $\mathrm{XII}^{\mathrm{e}}$ siècles, occupe le rez-de-cour d'un bâtiment dont les vestiges s'apparentent à ceux des maisons mixtes, courantes en Europe à cette période et largement documentées en Bretagne (Broes et al., 2013). Cet archétype de la maison médiévale présente un plan allongé et abrite sous un même toit hommes et animaux qui partagent parfois une même entrée. Les exemples bretons montrent généralement une séparation peu marquée des espaces sinon par la mise en place d'une cloison légère. La maison du Guildo ne s'en distingue que par sa taille, les maisons paysannes ne dépassant généralement pas une dizaine de mètres de longueur contre près du double ici. L'autre différence notable est la présence sur notre bâtiment d'un ou plusieurs étages traduisant la différenciation des fonctions domestiques et résidentielles. Les exemples de telles constructions en milieu castral restent rares sinon inconnus.

La place que l'on donne au cheval lors des importants travaux menés au milieu du $\mathrm{XV}^{\mathrm{e}}$ siècle participe certainement à la fonction de représentation que joue alors le château, occupé par des personnages de très haut rang ${ }^{\mathbf{4}}$, pour qui le cheval est un marqueur social fort (particulièrement à la fin du Moyen Âge, quand la chevalerie devient « un ordre d'élite de la noblesse », Flori, 1998, p. 266 et 269). Cet aspect est probablement d'autant plus vrai que les spécimens accueillis au château devaient être des bêtes d'exception : leur hébergement au château les valorise autant qu'ils valorisent celui-ci par leur présence. On peut voir dans l'exemple du Guildo les prémisses du traitement de l'écurie dans les châteaux de la Renaissance, qui, quand elles ne sont pas totalement invisibles, sont magnifiées et intégrées dans l'architecture d'apparat du château, en participant notamment à la monumentalisation de son entrée (Noblet, 2009, p. 129-130).

Les deux écuries aménagées dans les celliers à la fin $\mathrm{du} \mathrm{XV}^{\mathrm{e}}$ siècle puis à la fin du XVI ${ }^{\mathrm{e}}$ siècle ne participent plus de ce rôle symbolique et de représentation sociale. Leur rôle devient ou redevient purement utilitaire, en abritant des montures nécessaires pour les défenseurs du château. Ce pragmatisme est très perceptible dans le choix des lieux qui est fait pour aménager ces espaces : facilité d'accès, possibilité de loger le plus grand nombre possible de chevaux. Les aménagements réalisés restent légers. Même la construction du sol de galets lors des guerres de la Ligue reste modeste, réalisée à partir d'éléments pris à proximité immédiate du site. Il est toutefois notable que ces aménagements, ponctuels et temporaires, qui détournent des espaces de leur fonction première, suivent des principes ou des savoir-faire qui ne seront fixés qu'à partir du début du XVII ${ }^{\mathrm{e}}$ siècle dans les traités pour l'établissement d'écuries ${ }^{5}$ (Schwien et Jeannin, 2012). 


\section{Références bibliographiques}

AULIARD C., 1997, "Les maréchaux à l'époque médiévale : forgerons ou vétérinaires ?», Médiévales, n 33, p. 161-173.

Beuchet L., 2012, «Aux origines du château du Guildo (Côtes-d'Armor, France) », in L'origine du château médiéval, Château-Gaillard, 25, actes du colloque international de Rindern (Allemagne, 28 août-3 septembre 2010), Caen, Publications du CRAHM, p. 17-26.

BEUCHET L., à paraître, Les écuries du château $d u$ Guildo (Côtes-d'Armor), du XI e au XVI $I^{e}$ siècle.

Beuchet L., Picault C., 1998, « La forge du château du Guildo (Côtes-d'Armor), XIV - XV siècles ", in BECK P., L'innovation technique au Moyen Âge, Actes du VI congrès international d'archéologie médiévale, Paris, Errance ( Archéologie aujourd'hui »), p. 169-171

BourgeOIS L., 2009, Une résidence des comtes d'Angoulême autour de l'an Mil: le castrum d'Andone, Villejoubert, Charente. Publication des fouilles d'André Debord, 1971-1995, Caen, Publications du CRAHM, $560 \mathrm{p}$.

Broes F., Clavel V., ClercQ W. de, Fechner K. ROUPPERT V., VANMOERKERKE J., 2013, « À la recherche des espaces de stabulation. Étude pluridisciplinaire d'habitats du Néolithique au Moyen Âge dans le nord de la France ", Archéopages, 35, p. 6-16.

Contamine P., 1986, La guerre au Moyen Âge, Paris, PUF ( $2^{e}$ éd.), $516 \mathrm{p}$.
DietSCH-Sellami M.-F., 2000, Stock de grains carbonisés d'une salle basse du logis (XIV $-X V^{e}$ siècles) du château du Guildo (Côtes-d'Armor), Rapport d'étude dactylographié, Afan, Rennes, 8 p.

Dietsch-Sellami M.-F., 2003, Analyse carpologique d'une nouvelle cave du château du Guildo (Côtesd'Armor), Rapport d'étude dactylographié, Afan, Rennes, 6 p.

Dufour J.-Y., 2013, «Étables à bovins, écuries, bergeries, porcheries. Manuels agronomiques et vestiges médiévaux et modernes en Île-de-France », Archéopages, 35, p. 6o-66.

FLORI J., 1998, Chevaliers et chevalerie au Moyen Âge, Paris, Hachette littératures, $307 \mathrm{p}$.

LE Goff H., 2010, La Ligue en Bretagne : guerre civile et conflit international (1588-1598), Rennes, Presses universitaires de Rennes, $573 \mathrm{p}$.

LIÉVAUX P., 2005, Les écuries des châteaux français, Paris, Éditions du patrimoine, $303 \mathrm{p}$.

NoBLET J., 2009, « Les écuries des châteaux français à la Renaissance », in Franchet d'EsPÈrey P. ET Chatenet M. (dir), Les arts de l'équitation dans l'Europe de la Renaissance, Arles, Actes Sud, p. 118-137.

SCHWIEN J.-J., JEANNIN Y., 2012, « Loger, nourrir, équiper le cheval : un essai de synthèse pour la seconde partie du Moyen Âge dans l'est de la France et ailleurs", in LAZARIS S., Le cheval dans les sociétés antiques et médiévales, actes des journées d'étude internationales organisées par l'UMR 7044 (étude des civilisations de l'Antiquité), Strasbourg, 6-7 novembre 2009, Turnhout, Brepols ( «ibliothèque de l'Antiquité tardive », 22), p. 113-131, figures p. 240-271. 